\title{
Dephasing in Disordered Conductors due to Fluctuating Electric Fields
}

\author{
Axel Völker and Peter Kopietz \\ Institut für Theoretische Physik der Universität Göttingen, Bunsenstrasse 9, D-37073 Göttingen, \\ Germany
}

(June 18, 1999)

\begin{abstract}
We develop a novel eikonal expansion for the Cooperon to study the effect of space- and time-dependent electric fields on the dephasing rate of disordered conductors. For randomly fluctuating fields with arbitrary covariance we derive a general expression for the dephasing rate which is free of infrared divergencies in reduced dimensions. For time-dependent external fields with finite wavelength and sufficiently small amplitude we show that the dephasing rate is proportional to the square root of the electromagnetic power coupled into the system, in agreement with data by Wang and Lindelof [Phys. Rev. Lett. 59, 1156 (1987)].
\end{abstract}

PACS numbers: 72.15.Rn, 72.70.+m, 72.20.Ht, 73.23.-b

Typeset using REVTEX 


\section{INTRODUCTION}

The dephasing time $\tau_{\phi}$ of a particle in a quantum system is the time over which its wavefunction maintains phase coherence. For diffusive systems in dimensions $d=1$ and $d=2$ the dephasing time can be obtained experimentally from the weak-localization correction to the conductivity in a magnetic field. Recently Mohanty, Jariwala and Webb [1] used this method a measure $\tau_{\phi}$ as a function of the temperature $T$ in gold wires. They found that at sufficiently low temperatures $\tau_{\phi}$ approaches a finite value. This is in disagreement with the generally accepted point of view [2] that in thermal equilibrium $\tau_{\phi}$ should diverge as $T^{-p}$,

$p>0$, for $T \rightarrow 0$. A proposal that the observed saturation of $\tau_{\phi}$ is an intrinsic ground-state property of interacting electrons in a random potential [3] has been heavily criticized [4]. Until now, there is no general agreement on the microscopic mechanism for the observed saturation of $\tau_{\phi}$ at low temperatures.

One possible reason for this saturation might be external microwave radiation that is unintentionally coupled into the system [5]. According to Ref. [6] the dephasing rate $1 / \tau_{\mathrm{AC}}$ due to a time-dependent but spatially constant electric field is proportional to the microwave power $P$ absorbed by the system for small $P$, and crosses over to a $P^{1 / 5}$-law for larger $P$. Experimentally the effect of microwaves on weak localization has been studied by several authors [7 [10. Most of the data by Wang and Lindelof [7] can be fitted with $1 / \tau_{\mathrm{AC}} \propto P^{1 / 2}$. Below we shall offer a simple explanation for this behavior. More recent data by Webb et al. [10] are consistent with a $P^{1 / 5}$-law at high powers $P$, but in this experiment the microwaves seem to heat the sample, an effect which has not been taken into account in Ref. [6], and which we will neglect as well.

Following Altshuler et al. [6,11], we define the dephasing rate in the diffusive regime via the weak localization correction $\delta \sigma$ to the static conductivity. In the presence of an external electric field $\delta \sigma$ can be written as 6, 11, 12

$$
\delta \sigma=-\frac{\sigma_{0}}{\pi \nu_{d}} \int_{\tau_{\mathrm{el}}}^{\infty} d t \lim _{T_{0} \rightarrow \infty} \frac{1}{2 T_{0}} \int_{-T_{0}}^{T_{0}} d t_{0} \mathcal{C}\left(\mathbf{r}, \mathbf{r}, \frac{t}{2},-\frac{t}{2}, t_{0}\right)
$$


where $\sigma_{0}$ is the Drude conductivity, $\nu_{d}$ is the $d$-dimensional density of states, and $\tau_{\mathrm{el}}$ is the momentum relaxation time. The Cooperon $\mathcal{C}$ satisfies 1214

$$
\begin{aligned}
{\left[\partial_{t}+D \hat{\mathbf{P}}_{\mathbf{r}}^{2}+\Gamma_{0}+\right.} & \left.i\left[V\left(\mathbf{r}, t_{0}+t\right)-V\left(\mathbf{r}, t_{0}-t\right)\right]\right] \\
& \times \mathcal{C}\left(\mathbf{r}, \mathbf{r}^{\prime}, t, t^{\prime}, t_{0}\right)=\delta\left(\mathbf{r}-\mathbf{r}^{\prime}\right) \delta\left(t-t^{\prime}\right),
\end{aligned}
$$

where $\hat{\mathbf{P}}_{\mathbf{r}}=-i \nabla_{\mathbf{r}}$ is the momentum operator, $D$ is the diffusion coefficient, and the phenomenological cutoff $\Gamma_{0}$ describes dephasing due to processes that are not explicitly treated in our calculation, such as inelastic electron-phonon scattering or electron-electron scattering with energy-transfers $|\omega| \gtrsim T$. The potential $V(\mathbf{r}, t)$ is related to the longitudinal electric field via $e \mathbf{E}(\mathbf{r}, t)=\nabla_{\mathbf{r}} V(\mathbf{r}, t)$, where $-e$ is the charge of the electron. For $\Gamma_{0}=V=0$ the $t$-integration in Eq.(1) diverges in $d \leq 2$ at the upper limit, but for finite $\Gamma_{0}$ or $V$ the integration is cut off at some finite time, the so-called dephasing time.

\section{EIKONAL EXPANSION}

Formally Eq.(2) looks like the differential equation for the imaginary-time single-particle Green's function of an electron in a fluctuating external potential

$$
V_{t_{0}}(\mathbf{r}, t)=i\left[V\left(\mathbf{r}, t_{0}+t\right)-V\left(\mathbf{r}, t_{0}-t\right)\right]
$$

The real time version of this problem has been discussed extensively in the quantum field theory literature [15]. By means of a simple modification of the method developed by E. S. Fradkin [15] (see also Ref. [16]) we obtain the solution of Eq.(2) in the following form [12]

$$
\begin{aligned}
\mathcal{C}\left(\mathbf{r}, \mathbf{r}^{\prime}, t, t^{\prime}, t_{0}\right) & =\Theta\left(t-t^{\prime}\right) \int \frac{d \mathbf{k}}{(2 \pi)^{d}} e^{i \mathbf{k} \cdot\left(\mathbf{r}-\mathbf{r}^{\prime}\right)} \\
& \times e^{-\left(\Gamma_{0}+D \mathbf{k}^{2}\right)\left(t-t^{\prime}\right)-F\left(\mathbf{k}, t-t^{\prime} ; \mathbf{r}, t\right)}
\end{aligned}
$$

where the function $F$ satisfies the eikonal equation

$$
\begin{array}{r}
{\left[\partial_{\tau}+\partial_{t}+D\left(\hat{\mathbf{P}}_{\mathbf{r}}^{2}+2 \mathbf{k} \cdot \hat{\mathbf{P}}_{\mathbf{r}}\right)\right] F(\mathbf{k}, \tau ; \mathbf{r}, t)=} \\
V_{t_{0}}(\mathbf{r}, t)+D\left[\hat{\mathbf{P}}_{\mathbf{r}} F(\mathbf{k}, \tau ; \mathbf{r}, t)\right]^{2}
\end{array}
$$


with boundary condition $F(\mathbf{k}, 0 ; \mathbf{r}, t)=0$. Although in general Eq.(5) cannot be solved exactly, we can easily obtain an expansion of $F$ in powers of the potential $V_{t_{0}}(\mathbf{r}, t)$. Setting

$$
F(\mathbf{k}, \tau ; \mathbf{r}, t)=\sum_{n=1}^{\infty} F_{n}(\mathbf{k}, \tau ; \mathbf{r}, t)
$$

where $F_{n}$ involves by definition $n$ powers of $V_{t_{0}}$, successive terms may be calculated recursively [12,15, 16]. For our purpose we need only the first two terms. The linear term is

$$
\begin{aligned}
F_{1}(\mathbf{k}, \tau ; \mathbf{r}, t)= & \int \frac{d \mathbf{q} d \omega}{(2 \pi)^{d+1}} e^{i(\mathbf{q} \cdot \mathbf{r}-\omega t)} V_{t_{0}}(\mathbf{q}, \omega) \\
& \times \frac{1-e^{-\left[D\left(\mathbf{q}^{2}+2 \mathbf{k} \cdot \mathbf{q}\right)-i \omega\right] \tau}}{D\left(\mathbf{q}^{2}+2 \mathbf{k} \cdot \mathbf{q}\right)-i \omega}
\end{aligned}
$$

Here

$$
V_{t_{0}}(\mathbf{q}, \omega)=i\left[e^{-i \omega t_{0}} V(\mathbf{q}, \omega)-e^{i \omega t_{0}} V(\mathbf{q},-\omega)\right]
$$

is the Fourier transform of $V_{t_{0}}(\mathbf{r}, t)$, where

$$
V(\mathbf{q}, \omega)=\int d \mathbf{r} \int d t e^{-i(\mathbf{q} \cdot \mathbf{r}-\omega t)} V(\mathbf{r}, t)
$$

From Eq.(5) we find that the quadratic term is related to the linear one via

$$
F_{2}(\mathbf{k}, \tau ; \mathbf{r}, t)=-\int_{0}^{\tau} d \tau^{\prime} e^{\left(D \hat{\mathbf{P}}_{\mathbf{r}}^{2}+2 D \mathbf{k} \cdot \hat{\mathbf{P}}_{\mathbf{r}}+\partial_{t}\right)\left(\tau^{\prime}-\tau\right)} D\left[\hat{\mathbf{P}}_{\mathbf{r}} F_{1}\left(\mathbf{k}, \tau^{\prime} ; \mathbf{r}, t\right)\right]^{2}
$$

The explicit calculation yields

$$
\begin{aligned}
& F_{2}(\mathbf{k}, \tau ; \mathbf{r}, t)=\int \frac{d \mathbf{q}_{1} d \omega_{1}}{(2 \pi)^{d+1}} \int \frac{d \mathbf{q}_{2} d \omega_{2}}{(2 \pi)^{d+1}} \\
& \times e^{i\left[\left(\mathbf{q}_{1}+\mathbf{q}_{2}\right) \cdot \mathbf{r}-\left(\omega_{1}+\omega_{2}\right)\right] t} V_{t_{0}}\left(\mathbf{q}_{1}, \omega_{1}\right) V_{t_{0}}\left(\mathbf{q}_{2}, \omega_{2}\right) \\
& \times \\
& \times \frac{\left(D \mathbf{q}_{1} \cdot \mathbf{q}_{2}\right) e^{-\left[D\left(\left(\mathbf{q}_{1}+\mathbf{q}_{2}\right)^{2}+2 \mathbf{k} \cdot\left(\mathbf{q}_{1}+\mathbf{q}_{2}\right)\right)-i\left(\omega_{1}+\omega_{2}\right)\right] \tau}}{\left[D\left(\mathbf{q}_{1}^{2}+2 \mathbf{k} \cdot \mathbf{q}_{1}\right)-i \omega_{1}\right]\left[D\left(\mathbf{q}_{2}^{2}+2 \mathbf{k} \cdot \mathbf{q}_{2}\right)-i \omega_{2}\right]} \\
& \times\left\{\frac{e^{\left[D\left(\left(\mathbf{q}_{1}+\mathbf{q}_{2}\right)^{2}+2 \mathbf{k} \cdot\left(\mathbf{q}_{1}+\mathbf{q}_{2}\right)\right)-i\left(\omega_{1}+\omega_{2}\right)\right] \tau}-1}{D\left(\left(\mathbf{q}_{1}+\mathbf{q}_{2}\right)^{2}+2 \mathbf{k} \cdot\left(\mathbf{q}_{1}+\mathbf{q}_{2}\right)\right)-i\left(\omega_{1}+\omega_{2}\right)}\right. \\
& \quad+\frac{e^{2 D \mathbf{q}_{1} \cdot \mathbf{q}_{2} \tau}-1}{2 D \mathbf{q}_{1} \cdot \mathbf{q}_{2}}-\frac{e^{\left[D\left(\mathbf{q}_{1}^{2}+2 \mathbf{k} \cdot \mathbf{q}_{1}+2 \mathbf{q}_{1} \cdot \mathbf{q}_{2}\right)-i \omega_{1}\right] \tau}-1}{D\left(\mathbf{q}_{1}^{2}+2 \mathbf{k} \cdot \mathbf{q}_{1}+2 \mathbf{q}_{1} \cdot \mathbf{q}_{2}\right)-i \omega_{1}} \\
& \left.\quad-\frac{e^{\left[D\left(\mathbf{q}_{2}^{2}+2 \mathbf{k} \cdot \mathbf{q}_{2}+2 \mathbf{q}_{2} \cdot \mathbf{q}_{1}\right)-i \omega_{2}\right] \tau}-1}{D\left(\mathbf{q}_{2}^{2}+2 \mathbf{k} \cdot \mathbf{q}_{2}+2 \mathbf{q}_{2} \cdot \mathbf{q}_{1}\right)-i \omega_{2}}\right\} .
\end{aligned}
$$


Because we have made the diffusion approximation, the momentum-integrations in Eqs.(17,11) are restricted to $|\mathbf{q}|,\left|\mathbf{q}_{1}\right|,\left|\mathbf{q}_{2}\right|<1 / \ell \equiv 1 /\left(v_{F} \tau_{\mathrm{el}}\right)$, and the frequency-integrals to $|\omega|,\left|\omega_{1}\right|,\left|\omega_{2}\right|<1 / \tau_{\text {el }}$. Here $\ell$ is the elastic mean free path and $v_{F}$ is the Fermi velocity. For brevity we have not explicitly written out these cutoffs in the above expressions.

\section{DEPHASING DUE TO RANDOM FIELDS}

\section{A. General case}

We now assume that the potential $V(\mathbf{r}, t)$ is a random function with zero average and general covariance

$$
\left\langle V(\mathbf{q}, \omega) V\left(\mathbf{q}^{\prime}, \omega^{\prime}\right)\right\rangle=(2 \pi)^{d+1} \delta\left(\mathbf{q}+\mathbf{q}^{\prime}\right) \delta\left(\omega+\omega^{\prime}\right) g(\mathbf{q}, \omega)
$$

where $\langle\ldots\rangle$ denotes averaging over the probability distribution of $V$. The dephasing rate is then defined in terms of the average $\langle\delta \sigma\rangle$ of Eq.(四). Using the fact that after the averaging the Cooperon is independent of the time $t_{0}$, we obtain from Eqs.(1, 国)

$$
\langle\delta \sigma\rangle=-\frac{\sigma_{0}}{\pi \nu_{d}} \int_{\tau_{\mathrm{el}}}^{\infty} d t \int \frac{d \mathbf{k}}{(2 \pi)^{d}} e^{-\left(\Gamma_{0}+D \mathbf{k}^{2}\right) t-\Gamma(\mathbf{k}, t)}
$$

where

$$
\Gamma(\mathbf{k}, t)=-\ln \left\langle e^{-F(\mathbf{k}, t ; \mathbf{r}, t / 2)}\right\rangle
$$

We now perform a linked cluster expansion of $\Gamma(\mathbf{k}, t)$ in powers of the correlator $g(\mathbf{q}, \omega)$. To first order we find

$$
\Gamma(\mathbf{k}, t) \approx \Gamma_{1}(\mathbf{k}, t)+\Gamma_{2}(\mathbf{k}, t)
$$

where

$$
\begin{aligned}
& \Gamma_{1}(\mathbf{k}, t)=-\frac{1}{2}\left\langle F_{1}^{2}(\mathbf{k}, t ; \mathbf{r}, t / 2)\right\rangle \\
& \Gamma_{2}(\mathbf{k}, t)=\left\langle F_{2}(\mathbf{k}, t ; \mathbf{r}, t / 2)\right\rangle
\end{aligned}
$$


Introducing the notation $E_{\mathbf{k}}(\mathbf{q})=D\left(\mathbf{q}^{2}+2 \mathbf{k} \cdot \mathbf{q}\right)$ we obtain

$$
\begin{gathered}
\Gamma_{1}(\mathbf{k}, t)=\int \frac{d \mathbf{q} d \omega}{(2 \pi)^{d+1}} g(\mathbf{q}, \omega) \\
\times\left\{\frac{1-e^{-\left(E_{\mathbf{k}}(\mathbf{q})-i \omega\right) t}}{E_{\mathbf{k}}(\mathbf{q})-i \omega} \frac{1-e^{-\left(E_{\mathbf{k}}(-\mathbf{q})+i \omega\right) t}}{E_{\mathbf{k}}(-\mathbf{q})+i \omega}\right. \\
\left.-e^{-i \omega t} \frac{1-e^{-\left(E_{\mathbf{k}}(\mathbf{q})-i \omega\right) t}}{E_{\mathbf{k}}(\mathbf{q})-i \omega} \frac{1-e^{-\left(E_{\mathbf{k}}(-\mathbf{q})-i \omega\right) t}}{E_{\mathbf{k}}(-\mathbf{q})-i \omega}\right\}, \\
\Gamma_{2}(\mathbf{k}, t)=\int \frac{d \mathbf{q} d \omega}{(2 \pi)^{d+1}} g(\mathbf{q}, \omega) \frac{2 D \mathbf{q}^{2}}{E_{\mathbf{k}}(\mathbf{q})-i \omega} \\
\times\left\{\frac{1}{E_{\mathbf{k}}(-\mathbf{q})+i \omega}\left[t+\frac{1-e^{-2 D \mathbf{q}^{2} t}}{2 D \mathbf{q}^{2}}-\frac{1-e^{-\left(E_{\mathbf{k}}(\mathbf{q})-i \omega\right) t}}{E_{\mathbf{k}}(\mathbf{q})-i \omega}-\frac{1-e^{-\left(E_{\mathbf{k}}(-\mathbf{q})+i \omega\right) t}}{E_{\mathbf{k}}(-\mathbf{q})+i \omega}\right]\right. \\
\left.-\frac{1}{E_{\mathbf{k}}(-\mathbf{q})-i \omega}\left[\frac{\sin (\omega t)}{\omega}+e^{i \omega t}\left[\frac{1-e^{-2 D \mathbf{q}^{2} t}}{2 D \mathbf{q}^{2}}-\frac{1-e^{-\left(E_{\mathbf{k}}(\mathbf{q})+i \omega\right) t}}{E_{\mathbf{k}}(\mathbf{q})+i \omega}-\frac{1-e^{-\left(E_{\mathbf{k}}(-\mathbf{q})+i \omega\right) t}}{E_{\mathbf{k}}(-\mathbf{q})+i \omega}\right]\right]\right\} .
\end{gathered}
$$

\section{B. Nyquist noise}

As a special case let us assume that the potential $V$ is generated by equilibrium fluctua-

tions of the electric field due to the thermal motion of the electrons (Nyquist noise). Then $g(\mathbf{q}, \omega)$ is determined by the fluctuation-dissipation theorem, which implies [1]

$$
g(\mathbf{q}, \omega)=-f_{\mathbf{q}} \operatorname{coth}\left(\frac{\omega}{2 T}\right) \operatorname{Im} \epsilon^{-1}(\mathbf{q}, \omega)
$$

where $f_{\mathbf{q}}$ is the Fourier transform of the bare Coulomb interaction, and the dielectric function of the system is in the diffusive regime given by

$$
\epsilon(\mathbf{q}, \omega)=1+f_{\mathbf{q}} \nu_{d} \frac{D \mathbf{q}^{2}}{D \mathbf{q}^{2}-i \omega} .
$$

The corresponding dephasing rate in $d=1,2,3$ was calculated by Altshuler et al. [1] with the help of a Feynman path-integral representation of the solution of Eq.(2). It is instructive to reproduce the results of Ref. [11] within our eikonal expansion, treating $d$ as a continuous parameter. It is important to note that in the derivation of Eq.(2) the electromagnetic field is treated classically [1],14, so that our calculation takes only into account low-frequency 
Fourier components of the screened Coulomb interaction, with $|\omega| \lesssim T$. The correlator $g(\mathbf{q}, \omega)$ in Eqs.(18.19) can then be approximated by

$$
g(\mathbf{q}, \omega) \approx \Theta(T-|\omega|) \frac{2 T}{\nu_{d} D \mathbf{q}^{2}},
$$

Because the momentum integrals in the following analysis will be infrared and ultraviolet convergent, no further cutoffs are needed in our approach.

The dephasing rate is determined by the long-time behavior of $\Gamma(t) \equiv \Gamma(\mathbf{k}=0, t)$. In $d<2$ we find for $t \gg 1 / T$

$$
\Gamma(t) \sim C_{d} \frac{T t^{2-\frac{d}{2}}}{\nu_{d} D^{\frac{d}{2}}},
$$

where the numerical constant $C_{d}$ is given by

$$
C_{d}=\frac{2^{3-d}}{\pi^{\frac{d}{2}}(2-d)(4-d)} .
$$

Because $\Gamma(t)$ grows for large $t$ faster than linear, the term $\Gamma_{0} t$ in Eq.(13) is negligible, and the dephasing rate may be defined by $\Gamma\left(\tau_{\phi}\right)=1$, which yields

$$
\frac{1}{\tau_{\phi}}=\left[\frac{C_{d} T}{\nu_{d} D^{d / 2}}\right]^{\frac{2}{4-d}} .
$$

In $d=2$ we find for $t \gg 1 / T$

$$
\Gamma(t) \sim \frac{\ln (T t) T t}{2 \pi \nu_{2} D},
$$

so that $\tau_{\phi}$ satisfies

$$
\frac{1}{\tau_{\phi}}=\frac{\ln \left(T \tau_{\phi}\right) T}{2 \pi \nu_{2} D} .
$$

Keeping in mind that in a good metal $2 \pi \nu_{2} D \gg 1$, this implies to leading order

$$
\frac{1}{\tau_{\phi}}=\frac{\ln \left(2 \pi \nu_{2} D\right) T}{2 \pi \nu_{2} D},
$$

in agreement with Ref. [11]. Finally, in $d>2$ the long-time behavior of $\Gamma(t)$ is dominated by the term proportional to $t$ in the second line of Eq.(19). The total dephasing rate in $d>2$ can then be written as 


$$
\begin{aligned}
\frac{1}{\tau_{\phi}} & =\Gamma_{0}+\int \frac{d \mathbf{q} d \omega}{(2 \pi)^{d}} g(\mathbf{q}, \omega)\left[\frac{1}{\pi} \frac{D \mathbf{q}^{2}}{\left(D \mathbf{q}^{2}\right)^{2}+\omega^{2}}\right] \\
& =\Gamma_{0}+\tilde{C}_{d} T^{\frac{d}{2}} /\left(\nu_{d} D^{\frac{d}{2}}\right),
\end{aligned}
$$

where

$$
\tilde{C}_{d}=\frac{2^{2-d}}{\pi^{\frac{d}{2}}(d-2) \Gamma\left(\frac{d}{2}\right) \sin \left(\frac{d}{4} \pi\right)} .
$$

Note that for $d \rightarrow 2$ both prefactors $C_{d}$ and $\tilde{C}_{d}$ diverge as $\pi^{-1}|d-2|^{-1}$, signalling logarithmic corrections in $d=2$. The term in the braces of Eq.(29) is the dynamic structure factor of the diffusing electrons in the regime $|\omega| \lesssim T$, where the detailed balance factor $e^{-\omega / T}$ can be replaced by unity. This term agrees with the semiclassical dephasing rate derived by Chakravarty and Schmid [17], see also Ref [18]. It should be kept in mind, however, that Eq.(29) is only valid in $d>2$, where the integral is finite. Diagrammatically Eq.(29) ignores vertex corrections in the Bethe-Salpeter equation for the Cooperon, which become important in $d \leq 2$. In this case Eq.(29) should be replaced by the more general expressions (18, 19), which are free of infrared divergencies.

Our $\Gamma(t)$ corresponds precisely to the function $f_{d}(t)$ introduced in a recent preprint by Golubev and Zaikin [19]. To make contact with this work, let us assume that Eq.(2) remains valid for potentials $V(\mathbf{r}, t)$ due to Nyquist noise with frequencies in the range $|\omega|<1 / \tau_{\mathrm{el}}$, so that in Eqs.(18, 19) we may approximate $g(\mathbf{q}, \omega) \approx \Theta\left(\tau_{\mathrm{el}}^{-1}-|\omega|\right) \omega \operatorname{coth}\left(\frac{\omega}{2 T}\right) /\left(\nu_{d} D \mathbf{q}^{2}\right)$. Then we obtain in the quantum regime $T t \ll 1$ in quasi $1 d$ to leading order $\Gamma(t) \sim$ $\sqrt{2} \pi^{-1} \tau_{\mathrm{el}}^{-1 / 2} t /\left(\nu_{1} D^{\frac{1}{2}}\right)$, which agrees with the leading term in the expansion of the function $f_{1}(t)$ given in Eq.(28) of Ref. [19]. Moreover, also the subleading corrections to $f_{1}(t)$ given by Golubev and Zaikin [19] can be obtained within our eikonal expansion [12]. Hence, at least in $d=1$ we can completely reproduce the behavior of the function $f_{d}(t)$ discussed in Ref. 19] if we assume that the differential equation (2) for the Cooperon remains valid when the classical potential is replaced by a quantum field mediating the Coulomb interaction. This replacement has been claimed by Golubev and Zaikin to be consistent with a fully quantum mechanical calculation. It is the origin for the discrepancies between their work and Ref. 
[11].

\section{DEPHASING DUE TO EXTERNAL FIELDS}

In the experiments [7,10] microwaves are coupled into the system via an antenna attached to a suitable waveguide, such that alternating longitudinal currents are induced in the sample. Keeping in mind that the precise way in which the microwaves couple into the system is not known, we assume for simplicity that the electrons feel a longitudinal electric field of the form

$$
E(\mathbf{r}, t)=E_{0} \cos \left(\mathbf{q}_{0} \cdot \mathbf{r}-\omega_{0} t\right)
$$

where $\omega_{0}$ is the microwave frequency and the wave-vector $\mathbf{q}_{0}$ depends on the geometry of the waveguide and the antenna. The corresponding potential in Eq.(2) is

$$
V(\mathbf{r}, t)=V_{0} \sin \left(\mathbf{q}_{0} \cdot \mathbf{r}-\omega_{0} t\right)
$$

with $E_{0}=q_{0} V_{0} / e$. Let us emphasize that this is the total screened potential, which is the sum of the external potential and the induced potential. Of course, the Maxwell equation inside a metal contains a dissipative term, so that the field inside the metal is not given by a simple propagating wave [20]. In general we expect that the field distribution inside the metal depends on the boundary conditions and on the precise manner in which the microwaves are coupled into the system. Such a calculation is beyond the scope of this work. However, if the spatial variation of the field is sufficiently slow (i.e. $q_{0}$ is sufficiently small) the field inside the metal can still be approximated by a plane wave. To estimate the upper limit for $q_{0}$ where this approximation is correct, let us assume that the external potential applied to the electrons is $V^{\text {ext }}(\mathbf{r}, t)=V_{0}^{\text {ext }} \sin \left(\mathbf{q}_{0} \cdot \mathbf{r}-\omega_{0} t\right)$. The total potential is then

$$
V(\mathbf{r}, t)=V_{0}^{\mathrm{ext}} \operatorname{Im}\left[\epsilon^{-1}\left(\mathbf{q}_{0}, \omega_{0}\right) e^{i\left(\mathbf{q}_{0} \cdot \mathbf{r}-\omega_{0} t\right)}\right]
$$


where the longitudinal electric function in the diffusive regime is given in Eq.(21). Screening can be ignored in the regime where $\epsilon\left(\mathbf{q}_{0}, \omega_{0}\right)$ can be approximated by unity. From Eq.(21) it is easy to see that in $d=1$ this is the case when

$$
q_{0} \ll \sqrt{\frac{\omega_{0}}{D}}
$$

while in $d=2$ the external field is effectively not screened if

$$
q_{0} \ll \frac{\omega_{0}}{2 \pi e^{2} \nu_{2} D} .
$$

Keeping in mind that in a good metal $2 \pi e^{2} \nu_{2}>1 / \ell$, we see that Eqs.(34,35) together with $\omega_{0}<1 / \tau_{\text {el }}$ are more restrictive than the condition $q_{0}<1 / \ell$ which has to be satisfied in order to use the semiclassical Eq.(2). We assume that $q_{0}$ is sufficiently small so that the inequalities (34,35) are valid. From Eq.(8) we then obtain

$$
\begin{aligned}
V_{t_{0}}(\mathbf{q}, \omega) & =\frac{V_{0}^{\text {ext }}}{2}(2 \pi)^{d+1}\left[\delta\left(\omega-\omega_{0}\right)-\delta\left(\omega+\omega_{0}\right)\right] \\
& \times\left[e^{-i \omega_{0} t_{0}} \delta\left(\mathbf{q}-\mathbf{q}_{0}\right)+e^{i \omega_{0} t_{0}} \delta\left(\mathbf{q}+\mathbf{q}_{0}\right)\right] .
\end{aligned}
$$

This expression is now inserted into the general results given in Eqs.(7) and (11). For $F_{1}$ we get

$$
\begin{aligned}
& F_{1}(\mathbf{k}, \tau ; \mathbf{r}, t)=-i \frac{e E_{0}}{q_{0}}\left\{e^{i\left(\mathbf{q}_{0} \cdot \mathbf{r}-\omega_{0} t_{0}\right)} \operatorname{Im}\left(\frac{1-e^{-D\left(\mathbf{q}_{0}^{2}+2 \mathbf{k} \cdot \mathbf{q}_{0}\right) \tau} e^{i \omega_{0} \tau}}{D\left(\mathbf{q}_{0}^{2}+2 \mathbf{k} \cdot \mathbf{q}_{0}\right)-i \omega_{0}} e^{-i \omega_{0} t}\right)\right. \\
&\left.+e^{-i\left(\mathbf{q} 0 \cdot \mathbf{r}-\omega_{0} t_{0}\right)} \operatorname{Im}\left(\frac{1-e^{-D\left(\mathbf{q}_{0}^{2}-2 \mathbf{k} \cdot \mathbf{q}_{0}\right) \tau} e^{i \omega_{0} \tau}}{D\left(\mathbf{q}_{0}^{2}-2 \mathbf{k} \cdot \mathbf{q}_{0}\right)-i \omega_{0}} e^{-i \omega_{0} t}\right)\right\} .
\end{aligned}
$$

Performing the analogous calculation for the function $F_{2}$ given in Eq.(11) would result in a rather lengthy expression. Since in the following we are only interested in the leading terms of an expansion in powers of $q_{0}$, it is more convenient to first expand $F_{1}$ and then use Eq.(10) to obtain $F_{2}$.

If we take the limit $\mathbf{q}_{0} \rightarrow 0$ keeping $E_{0}$ constant we obtain a spatially constant field, which has been considered in Ref. [6]. In this limit the microwaves do not affect the density of the electrons. For finite $q_{0}$, however, the electric field induces a density modulation with amplitude $\rho_{0}=E_{0} q_{0} /(4 \pi)$. We now show that for sufficiently small microwave power $P$ this 
leads to a dephasing rate $1 / \tau_{\mathrm{AC}}$ proportional to $P^{1 / 2}$, as observed in Ref. [7]. Note that the microwave power $P$ coupled into the sample can be estimated as [5, 10]

$$
P=\frac{\left(E_{0} L\right)^{2}}{2 R_{\mathrm{tot}}} \propto E_{0}^{2},
$$

where $L$ is the effective sample length including leads, and $R_{\text {tot }}$ is the total resistance.

According to Eqs.(11, (1) the weak localization correction $\delta \sigma$ is determined by $F(\mathbf{k}, t ; \mathbf{r}, t / 2)$.

In the experiments [7, [10] the wavelength of the microwaves is larger than the size of the sample, so that we may expand $F(\mathbf{k}, t ; \mathbf{r}, t / 2)$ in powers of $q_{0}$. From Eq.(37) we obtain

$$
\begin{aligned}
& F_{1}(\mathbf{k}, t ; \mathbf{r}, t / 2)=2 D e E_{0} \omega_{0}^{-2} g_{1}\left(\omega_{0} t\right)\left\{2 k_{\|} \sin \left(\omega_{0} t_{0}\right)\right. \\
& \left.\quad+q_{0} \cos \left(\omega_{0} t_{0}\right)\left[i\left(1-2 D k_{\|}^{2} t\right)+2 r_{\|} k_{\|}\right]\right\}+O\left(E_{0} q_{0}^{2}\right) .
\end{aligned}
$$

where $k_{\|}=\mathbf{k} \cdot \mathbf{q}_{0} / q_{0}, r_{\|}=\mathbf{r} \cdot \mathbf{q}_{0} / q_{0}$ and

$$
g_{1}(x)=x \cos \left(\frac{x}{2}\right)-2 \sin \left(\frac{x}{2}\right) .
$$

Inserting this result into Eq.(10) yields

$$
\begin{aligned}
F_{2}(\mathbf{k}, t ; \mathbf{r}, t / 2) & =4 D\left(e E_{0}\right)^{2} \omega_{0}^{-3} g_{2}\left(\omega_{0} t\right) \sin ^{2}\left(\omega_{0} t_{0}\right) \\
& +O\left(E_{0}^{2} q_{0}\right)
\end{aligned}
$$

with

$$
g_{2}(x)=x+\frac{x}{2} \cos (x)-\frac{3}{2} \sin (x) .
$$

Note that the second line in Eq.(39) is the leading correction for finite $q_{0}$ and small $E_{0}$. It can be shown [12] that in the limit $q_{0} \rightarrow 0$ all higher order terms $F_{n}, n \geq 3$ in our eikonal expansion vanish, so that the exact solution of the eikonal equation (5) is given by $F_{q_{0}=0}=\left[F_{1}+F_{2}\right]_{q_{0}=0}$. The fact that the Cooperon in a spatially constant field can be calculated exactly is obvious in a gauge where the electric field is represented in terms of a vector potential [6]. It is reassuring to see that our eikonal expansion reproduces the exact solution in a different gauge. Substituting Eqs.(39,41) into Eq.(14) and performing the k-integration, we obtain for the weak localization correction to the static conductivity 


$$
\begin{aligned}
\delta \sigma & =-\frac{\sigma_{0}}{\pi \nu_{d}} \frac{\omega_{0}^{\frac{d}{2}-1}}{(4 \pi D)^{d / 2}} \int_{\omega_{0} \tau_{\mathrm{el}}}^{\infty} \frac{d x}{x^{d / 2}} e^{-\alpha g_{3}(x)-\gamma x} \\
& \times \frac{1}{\pi} \int_{0}^{\pi} d \varphi \frac{e^{\alpha g_{3}(x) \cos (2 \varphi)-i \beta g_{1}(x) \cos \varphi}}{\sqrt{1-2 i \beta g_{1}(x) \cos \varphi}} .
\end{aligned}
$$

where

$$
g_{3}(x)=x+\sin x-\frac{8}{x} \sin ^{2}\left(\frac{x}{2}\right)
$$

and we have introduced the dimensionless parameters

$$
\begin{aligned}
& \alpha=\frac{D\left(e E_{0}\right)^{2}}{\omega_{0}^{3}}, \\
& \beta=\frac{2 D\left|e E_{0}\right| q_{0}}{\omega_{0}^{2}}, \\
& \gamma=\frac{\Gamma_{0}}{\omega_{0}} .
\end{aligned}
$$

Note that after the $\varphi$-integration the integral in Eq. (43) is real, so that we may replace the integrand by its real part, which is independent of the sign of $E_{0}$. This is the reason why in the definition (46) of $\beta$ only the absolute value of $E_{0}$ appears. Note that $\beta$ is proportional to the absolute value of the amplitude $\rho_{0}=E_{0} q_{0} /(4 \pi)$ of the density wave associated with the longitudinal electric field.

The order of magnitude of the non-equilibrium dephasing rate $1 / \tau_{\mathrm{AC}}$ can be estimated as $1 / \tau_{\mathrm{AC}} \approx \omega_{0} / x_{c}$, where $x_{c}$ is the effective large- $x$ cutoff for the integration in Eq.(43). For simplicity we now set $\gamma=0$. Depending on the ratio $\alpha / \beta=\left|e E_{0}\right| /\left(2 \omega_{0} q_{0}\right)=\left|V_{0}\right| /\left(2 \omega_{0}\right)$, we obtain different behaviors for $1 / \tau_{\mathrm{AC}}$. In the limit $\alpha / \beta \rightarrow \infty$ we recover the results of Ref. [6]. In this case $1 / \tau_{\mathrm{AC}} \propto \omega_{0} \alpha$ for $\alpha \ll 1$, and $1 / \tau_{\mathrm{AC}} \propto \omega_{0} \alpha^{1 / 5}$ for $\alpha \gg 1$. Note that $\alpha$ is proportional the microwave power $P \propto E_{0}^{2}$ absorbed by the system, while $\beta$ is proportional to $P^{1 / 2}$. Obviously, for sufficiently small $P$ we always have $\beta \gg \alpha$. Then the cutoff for the $x$-integration in Eq. (43) is determined by $\beta$, and we can set $\alpha=0$ to determine $x_{c}$. The $x$-integration is effectively cut of where the integrand starts to oscillate. Thus we estimate $x_{c}$ from the condition $\beta g_{1}\left(x_{c}\right) \approx 1$. For $\beta \ll 1$ the effective cutoff $x_{c}$ is large compared with 
unity, so that with Eq. (44) we obtain $1 / x_{c} \propto \beta$. This implies

$$
\frac{1}{\tau_{\mathrm{AC}}} \propto \omega_{0} \beta, \text { for } \alpha \ll \beta \ll 1
$$

On the other hand, for $\beta \gg 1$ the $x$-integration is cut off at small $x$ and we approximate $g_{1}(x) \approx-\frac{x^{3}}{12}$. This yields

$$
\frac{1}{\tau_{\mathrm{AC}}} \propto \omega_{0} \beta^{1 / 3}, \text { for } \alpha \ll \beta \text { and } \beta \gg 1
$$

Hence, for sufficiently small $P$ the dephasing rate $1 / \tau_{\mathrm{AC}}$ is proportional to $P^{1 / 2}$. Moreover, in this regime $\omega_{0} / \tau_{\mathrm{AC}}$ should be independent of $\omega_{0}$ as long as the dispersion of the longitudinal density wave (i.e. the dependence of $q_{0}$ on $\omega_{0}$ ) can be neglected. Note that the condition $\beta \gg \alpha \gg \gamma$ where $1 / \tau_{\mathrm{AC}}$ should exhibit a $P^{1 / 2}$-dependence can also be written as

$$
D q_{0}^{2} \gg \frac{\omega_{0}}{\left|V_{0}\right|} \Gamma_{0} \gg \Gamma_{0}
$$

Thus, for finite $\Gamma_{0}$ the value of $q_{0}$ must be sufficiently large to observe the $P^{1 / 2}$-law. Keeping in mind that according to the conventional point of view [4 6] the intrinsic dephasing rate $\Gamma_{0}$ should vanish for $T \rightarrow 0$, we conclude that at sufficiently low temperatures and small $V_{0}$ Eq.(50) can be satisfied for experimentally relevant wave-vectors $q_{0}$. Note that according to Ref. [5] the observed saturation of the dephasing time, which is typically of the order of a few nanoseconds, is due to some external noise. If this is correct, then the experimentally observed saturation value of the dephasing rate should not be identified with $\Gamma_{0}$. The same arguments apply for the condition $\left|V_{0}\right| \gg \Gamma_{0}$ that follows from Eq.(50) together with Eqs.34.35). For low enough temperatures this inequality should always be satisfied. (Most recent experimental data [22] indicates $\Gamma_{0} \rightarrow 0$ for $T \rightarrow 0$ in narrow Ag wires. These probes therefore seem to be suiting to test our findings.) In conclusion, in the limit $T \rightarrow 0$, the only relevant restriction following from Eq.(50) should be $\left|V_{0}\right| \ll \omega_{0}$.

For convenience, let us summarize the assumptions made in our derivation of the dephasing time due to external microwave radiation in this section. First of all, we have assumed that the microwave field inside the metal can be approximated by a propagating wave with 
wave-vector $\mathbf{q}_{0}$, frequency $\omega_{0}$ and amplitude $E_{0}=q_{0} V_{0} / e$. We have argued that this approximation is valid in a regime where the dielectric function of the bulk system can be approximated by unity, which is possible if $q_{0} \ll \sqrt{\omega_{0} / D}$ in $d=1$, and $q_{0} \ll \omega_{0} /\left(2 \pi e^{2} \nu_{2} D\right)$ in $d=2$. Throughout this work we have assumed diffusive dynamics and calculated the weak localization correction to the conductivity from the semiclassical equation (2), which is of course only correct for sufficiently small wave-vectors $\left(q_{0}<1 / \ell\right)$ and frequencies $\left(\omega_{0}<1 / \tau_{\text {el }}\right)$. Note that the latter inequality sets the upper limit for the microwave frequency where our approach remains valid. If these restrictions are satisfied, then the dephasing rate due to microwaves is

$$
\frac{1}{\tau_{\mathrm{AC}}} \propto\left\{\begin{array}{ll}
\omega_{0}^{2 / 5} P^{1 / 5} & \text { for }\left|V_{0}\right| \gg \omega_{0} \text { and } D\left(e E_{0}\right)^{2} \gg \omega_{0}^{2}, \\
\omega_{0}^{-2} P & \text { for }\left|V_{0}\right| \gg \omega_{0} \text { and } D\left(e E_{0}\right)^{2} \ll \omega_{0}^{2}, \\
\left(q_{0} \omega_{0}\right)^{1 / 3} P^{1 / 6} & \text { for }\left|V_{0}\right| \ll \omega_{0} \text { and } D\left|e E_{0}\right| q_{0} \gg \omega_{0}^{2} \\
q_{0} \omega_{0}^{-1} \sqrt{P} & \text { for }\left|V_{0}\right| \ll \omega_{0} \text { and } D\left|e E_{0}\right| q_{0} \ll \omega_{0}^{2}
\end{array},\right.
$$

where $P \propto E_{0}^{2}$ is the microwave-power absorbed by the system, and $V_{0}=e E_{0} / q_{0}$.

\section{COMPARISON WITH EXPERIMENTS AND SUMMARY}

Some time ago Wang and Lindelof [7] have measured $1 / \tau_{\mathrm{AC}}$ as a function of $P$ in magnesium films. Their data from Ref.[7(a)] are reproduced in Fig.11. At $\omega_{0} / 2 \pi=0.66 \mathrm{GHz}$ our prediction $\omega_{0} / \tau_{\mathrm{AC}} \propto P^{1 / 2}$ is in good agreement with the experiment. Although the data at $\omega_{0} / 2 \pi=3.61 \mathrm{GHz}$ cannot be fitted by a straight line through the origin, the data roughly scale as $1 / \tau_{\mathrm{AC}} \propto \omega_{0}^{-1}$ for fixed and small $P$. One should keep in mind, however, that in the experiments [7] the precise value of the microwave power coupled into the system was not measured, and the power axis for the two sets of data was rescaled differently. Further evidence for the $P^{1 / 2}$-law can be found in Fig.19 of Ref.[7(b)].

Recent measurements of $1 / \tau_{\mathrm{AC}}$ by Webb et al. [10] suggest a $P^{1 / 5}$-law for large $P$ in a limited range of frequencies. Our calculation shows that the $P^{1 / 5}$-law should hold as long as $\alpha \gg \max \{\beta, 1\}$, while for $\beta \gg \max \{\alpha, 1\}$ we predict $1 / \tau_{\mathrm{AC}} \propto \beta^{1 / 3} \propto P^{1 / 6}$. Keeping in 
mind that $\alpha / \beta \propto \omega_{0}^{-1}$ we predict a crossover from a $P^{1 / 5}$ - via a $P^{1 / 6}$ - to a $P^{1 / 2}$-behavior as the frequency is increased. The data shown in Fig.6 of Ref. [10] are consistent with the existence of such a crossover.

In summary, by means of an eikonal expansion for the Cooperon in a slowly varying scalar potential we have derived a general expression for the dephasing rate due to fluctuating electric fields in disordered metals. Our method is a physically transparent alternative to the path-integral approach used in Ref. [11]. For randomly fluctuating fields with zero average and arbitrary covariance the dephasing rate can be obtained from Eqs.(18,19). These expressions take vertex corrections into account and remain finite in reduced dimensions, where the well-known [17,18] semiclassical result Eq.(29) is infrared divergent for some physically relevant $g(\mathbf{q}, \omega)$. For example, in the case of $1 / f$-noise $18 \|$, where $g(\mathbf{q}, \omega) \propto 1 / \omega$, the integral in Eq.(29) is divergent in $d \leq 2$, while our more general result $(18,19)$ is finite. We have also studied dephasing due to external electric fields, and have proposed an explanation for the data of Ref. [7]. Finally, we point out that our eikonal method can also be used to calculate the Diffuson in a fluctuating electric field, which is of current interest in several contexts [21].

\section{ACKNOWLEDGMENTS}

We thank P. E. Lindelof and P. Mohanty for their comments and for helping us to understand the experiments [0, 10]. This work was supported by the DFG via the Heisenberg Programm and SFB 345. 


\section{REFERENCES}

[1] P. Mohanty, E. M. Jariwala, and R. A. Webb, Phys. Rev. Lett. 78, 3366 (1997).

[2] See, for example, Y. Imry, Introduction to Mesoscopic Physics, (Oxford University Press, Oxford, 1997).

[3] P. Mohanty and R. A. Webb, Phys. Rev. B 55, R13452 (1997); D. S. Golubev and A. D. Zaikin, Phys. Rev. Lett. 81, 1074 (1998); Phys. Rev. B 59, 9195 (1999).

[4] I. L. Aleiner, B. L. Altshuler, and M. E. Gershenson, Phys. Rev. Lett. 82, 3190 (1999); cond-mat/9808053.

[5] B. L. Altshuler, M. E. Gershenson, and I. Aleiner, Physica E 3, 58 (1998).

[6] B. L. Altshuler, A. G. Aronov, and D. E. Khmelnitskii, Solid State Commun. 39, 619 (1981).

[7] S. Wang and P. E. Lindelof, (a) Phys. Rev. Lett. 59, 1156, (1987); (b) J. Low Temp. Phys. 71, 403 (1988).

[8] S. A. Vitkalov et al., Zh. Eksp. Teor. Fiz. 94, 376 (1988) [Sov. Phys. JETP 67, 1080 (1988)]

[9] J. Liu and N. Giordano, Phys. Rev. B 43, 1385 (1991).

[10] R. A. Webb et al., in Quantum Coherence and Decoherence, edited by K. Fujikawa and Y. A. Ono, (North Holland, 1998).

[11] B. L. Altshuler, A. G. Aronov, and D. E. Khmelnitskii, J. Phys. C 15, 7367 (1982); B. L. Altshuler and A. G. Aronov in Electron-Electron Interactions in Disordered Systems, edited by A. L. Efros and M. Pollak (North Holland, Amsterdam, 1985).

[12] A. Völker and P. Kopietz, in preparation.

[13] Note that the time-labels of the potentials in Eq.(2) are $t_{0} \pm t$, and not $t_{0} \pm t / 2$, as 
incorrectly given in Refs. [6, 11, 14]. This error has first been pointed out by W. Eiler, J. Low Temp. Phys. 56, 481 (1984).

[14] M. Vavilov and V. Ambegaokar, cond-mat/9902127.

[15] A. V. Svidzinskii, Zh. Eksp. Teor. Fiz. 31, 324 (1957) [Sov. Phys. JETP 4, 179 (1957)]; B. M. Barbashov, Zh. Eksp. Teor. Fiz. 48, 607 (1965) [Sov. Phys. JETP 21, 402 (1965)]; E. S. Fradkin, Nucl. Phys. 76, 588 (1966); V. N. Popov, Functional Integrals in Quantum Field Theory and Statistical Physics, (Reidel, Dordrecht, 1983).

[16] P. Kopietz, Int. J. Mod. Phys. B 10, 2111 (1996).

[17] S. Chakravarty and A. Schmid, Phys. Rep. 140, 193 (1986).

[18] Y. Imry, H. Fukuyama, and P. Schwab, Europhys. Lett. 47, 608 (1999).

[19] D. S. Golubev and A. D. Zaikin, cond-mat/9907494.

[20] See, for example, J. M. Ziman, Principles of the Theory of Solids, 2nd Edition, (Cambridge University Press, Cambridge, 1972).

[21] F. von Oppen and A. Stern, Phys. Rev. Lett. 79, 1114 (1997); D. G. Polyakov and K. V. Samokhin, Phys. Rev. Lett. 80, 1509 (1998); R. Raimondi, P. Schwab, and C. Castellani, Phys. Rev. B 60, 5818 (1999); M. Leadbeater et al., cond-mat/9905240.

[22] A.B. Gougam et al., cond-mat/9912137 


\section{FIGURES}

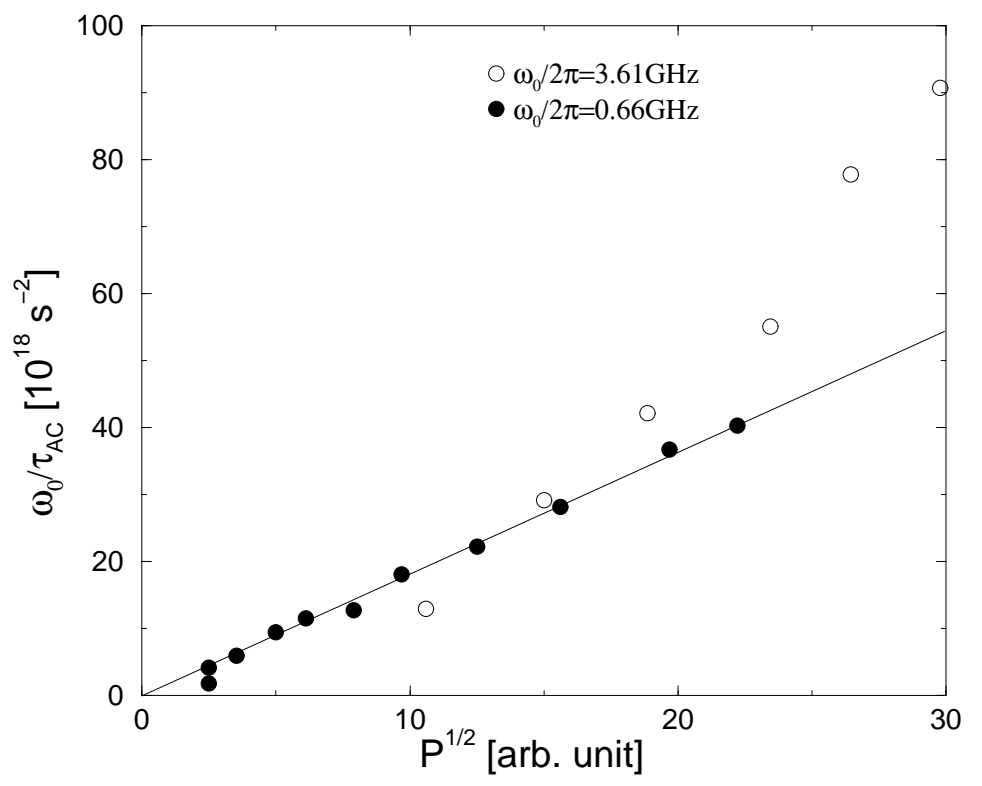

FIG. 1. Data for $\omega_{0} / \tau_{\mathrm{AC}}$ as function of $P^{1 / 2}$ from Fig.3 of Ref.[7(a)]. Here $P$ is the microwave power coupled into the system. The solid line is a fit of the data at $\omega_{0} / 2 \pi=0.66 \mathrm{GHz}$ to our prediction $\omega_{0} / \tau_{\mathrm{AC}} \propto P^{1 / 2}$, which is valid for small $P$. 